\title{
Integration on Supermanifolds and a Generalized Cartan Calculus
}

\author{
R. F. Picken and K. Sundermeyer \\ Institut für Theorie der Elementarteilchen, Freie Universität Berlin, FB20, WE4 Arnimallee 14, \\ D-1000 Berlin 33
}

\begin{abstract}
A suggestion by Berezin for a method of integration on supermanifolds is given a precise differential geometric meaning by assuming that a supermanifold is the total space of a fibre bundle with connection. The relevant objects for integration are identified as suitable horizontal/vertical projections of hyperforms. The latter are generalizations of differential forms having both covariant and contravariant indices. The exterior calculus of these projected hyperforms is developed, analogously to the Cartan calculus, by introducing appropriate derivations and determining their commutators, respectively anticommutators.
\end{abstract}

\section{Introduction}

The concepts of rigid and curved superspace have turned out to be of great importance in current research on supersymmetry and supergravity. As originally introduced by Salam and Strathdee [1] superspace has, besides the coordinates $x^{\mu}$ $(\mu=0, \ldots, 3)$, which are commuting (even, bosonic), additional anticommuting (odd, fermionic) coordinates $\theta^{\alpha}(\alpha=1, \ldots, 4)$. Superfields are functions depending on these variables and encode both bosonic and fermionic fields by means of a Taylor expansion in the odd variables. Integration of superfields with respect to the odd variables is given an operational definition by the Berezin integration rules [2]. Also a (super)-tensor calculus and the notions of (super)-connection, -torsion and -curvature are used frequently in the physics literature $[3,4]$.

Many authors have investigated how to make these more or less heuristic ideas mathematically rigorous. Rogers [5] introduced the concept of a $D_{0}+D_{1}$ dimensional supermanifold modelled over $B_{L}^{\left(D_{0}, D_{1}\right)}$, a space obtained from a Grassmann algebra $B_{L}$. Several modifications of her approach have been proposed $[4,6-8]$. The construction of tensor bundles on supermanifolds broadly resembles the procedure for $C^{\infty}$ manifolds.

What is still lacking is a fully satisfactory theory of integration on supermanifolds mimicking the Berezin integration rules. For $C^{\infty}$ manifolds the relevant 
objects for an integration theory are differential forms. This is not true for supermanifolds. In his last article Berezin [9] pointed out, that in this case one must consider more general tensors with both covariant and contravariant components (which we shall call hyperforms in the sequel). Although he was able to define a consistent supermanifold integral, his definition uses an ad hoc recipe for which we shall give a geometric interpretation. By this means we arrive at a truly geometric, chart-independent integration theory.

In Sect. 2 we make some informal remarks on superspace and supermanifolds. We would like to point out that superspace $B_{L}^{(4,4)}$ is not yet rigid superspace in physicists' jargon. Rigid superspace is a manifold modelled over $B_{L}^{(4,4)}$, but with more structure. This is to be compared with Minkowski space, which is a quasiRiemannian manifold modelled over $\mathbb{R}^{4}$ with the Poincare group as isometry group. Similarly a $G^{\infty}$ function is not the same as a superfield, of which one demands that it transforms according to some representation of the graded Poincaré group. These additional structures are however of no relevance for our arguments.

In Sect. 3 we discuss integration on supermanifolds. The first part of this section is largely a repetition of Berezin's arguments [9] (see also [10-12]), about why one needs hyperforms. Berezin's proposal for a supermanifold integration contains, in our view, a non-geometric ingredient. We overcome this by assuming that the supermanifold is the total space of a bundle with connection. The volume form turns out to be a $P$-hyperform constructed out of suitable pieces of the horizontal and vertical tangent and cotangent spaces of the bundle.

After having identified $P$-hyperforms as the relevant objects for integration on $G^{\infty}$ supermanifolds (comparable to differential forms for $C^{\infty}$ manifolds), we improve in Sect. 4 on the previous coordinate-based formulation of Sect. 3. $P$-hyperforms are now obtained from hyperforms by a projection.

Next we aim at an exterior calculus for $P$-hyperforms. We arrive at it in two steps: first in Sect. 5 we develop an exterior calculus for hyperforms, then in Sect. 6 we "project" this onto $P$-hyperforms. The operations are an exterior product $x$, an exterior derivative $\mathrm{d}$, contractions with respect to a vector field $i_{X}$, and derivations obtained from $\mathrm{dl}$ and $i_{X}$ by taking suitable commutators or anticommutators. The exterior derivative $\mathrm{d} l$ is a covariant derivative acting on vector-valued forms. On the space of $P$-hyperforms we can also define a Hodge duality operation.

In our conclusions in Sect. 7 we briefly discuss a possible enrichment of the exterior calculus on $P$-hyperforms by adding further derivations. Finally, since this article is mainly intended to be mathematical, we merely indicate why and how the calculus may be applied to supersymmetric field theories, leaving further details for future articles.

\section{Superspace and Supermanifolds}

The supermanifolds we are dealing with are modelled over flat superspace $B_{L}^{\left(D_{0}, D_{1}\right)}$, the Cartesian product of $D_{0}$ copies of $B_{L, 0}$ and $D_{1}$ copies of $B_{L, 1}$, where $B_{L, 0}$ and $B_{L, 1}$ are the even, respectively odd, subspaces of a real Grassmann algebra $B_{L}$ (with $L$ anticommuting generators). Functions from $B_{L}^{\left(D_{0}, D_{1}\right)}$ to $B_{L}$ will be taken to be $G^{\infty}$, 
i.e. infinitely differentiable with respect to all arguments, which in turn implies that the function admits a finite Taylor series expansion in the odd arguments, with infinitely differentiable functions of the even arguments as coefficients [5]. A $\left(D_{0}+D_{1}\right)$-dimensional supermanifold $M^{\left(D_{0}, D_{1}\right)}$ is constructed from $B_{L}^{\left(D_{0}, D_{1}\right)}$ in the usual way by means of an atlas of charts $\bigcup_{i \in I}\left(U_{i}, \varphi_{i}\right)$ with $U_{i}$ an open cover of $M^{\left(D_{0}, D_{1}\right)}$ and a homeomorphism $\varphi_{i}$ of $U_{i}$ onto an open subset of $B_{L}^{\left(D_{0}, D_{1}\right)}$. If the overlap $U_{i} \cap U_{j}$ is non-empty we require the transition function $\varphi_{j} \circ \varphi_{i}^{-1}$ to be $G^{\infty}$.

A chart map $\varphi$ induces coordinates $\varphi^{M}(m)=z^{M}=\left\{x^{\mu}, \theta^{\alpha}\right\}\left(M=1, \ldots, D_{0}+D_{1}\right.$, $\left.\mu=1, \ldots, D_{0}, \alpha=1, \ldots, D_{1}\right)$ for $m \in U$. If we change to other coordinates we require this change to respect evenness/oddness in the following sense: if $(X)$ denotes the grading of a Grassmann element $X$, i.e. $(X)=0$ if $X$ is even and $(X)=1$ if $X$ is odd, then under a coordinate change $z^{M} \rightarrow \bar{z}^{M}$ we require $\left(z^{M}\right)=\left(\bar{z}^{M}\right)$.

Equipped with the notion of differentiability in $B_{L}^{\left(D_{0}, D_{1}\right)}$ one can construct the tangent bundle $T M^{\left(D_{0}, D_{1}\right)}$. At a point $m \in U \subset M^{\left(D_{0}, D_{1}\right)}$ the tangent space $\underset{(m)}{T} M^{\left(D_{0}, D_{1}\right)}$ is spanned (in a coordinate basis) by $\left\{{ }_{M} \partial=\frac{\vec{\partial}}{\partial z^{M}}\right\}$ (we use the de Witt [4] conventions for index manipulation, which conveniently avoid factors of $(-1)$ ). The dual space to $\underset{(m)}{T} M^{\left(D_{0}, D_{1}\right)}$ denoted $\underset{(m)}{T^{*}} M^{\left(D_{0}, D_{1}\right)}$ is spanned by $\left\{d z^{M}\right\}$, where $\left\langle{ }_{N} \partial \mid d z^{M}\right\rangle={ }_{N} \delta^{M}$ (the Kronecker delta). This is in turn gives rise to the cotangent bundle $T^{*} M$. In general tensor fields of type $(p, r)$ are elements of $\otimes^{p} T^{*} M^{\left(D_{0}, D_{1}\right)} \otimes \otimes^{r} T M^{\left(D_{0}, D_{1}\right)}$.

The components of a tensor of type $(p, r)$ are displayed in a coordinate basis as:

$$
d z^{N_{1}} \otimes \ldots \otimes d z^{N_{p}}\left({ }_{N_{p} \ldots N_{1}} T^{M_{1} \ldots M_{r}}\right) \otimes_{M_{r}} \partial \otimes \ldots \otimes{M_{1}}_{1} \partial .
$$

In the following sections we will need tensors with special symmetry properties, which generalize the differential forms of ordinary differential geometry. This subspace of $(p, r)$ tensors is denoted $\Lambda_{p}^{r}\left(M^{\left(D_{0}, D_{1}\right)}\right)$ and is spanned by

$$
d z^{N_{1}} \wedge \ldots \wedge d z^{N_{p}} \otimes_{M_{r}} \partial \vee \ldots \vee{ }_{M_{1}} \partial
$$

where $\wedge$ is the graded antisymmetric wedge product, and

$$
d z^{N} \wedge d z^{M}=d z^{[N} \wedge d z^{M)}=-(-1)^{(N)(M)} d z^{M} \wedge d z^{N},
$$

where $(N)=\left(d z^{N}\right)$, and $\vee$ is the graded symmetric product

$$
{ }_{N} \partial \vee{ }_{M} \partial={ }_{(N} \partial \vee{ }_{M]} \partial=+(-1)^{(N)(M)}{ }_{M} \partial \vee_{N} \partial \text {. }
$$

We will call an element of $\Lambda_{p}^{r}\left(M^{\left(D_{0}, D_{1}\right)}\right)$ a $(p, r)$ hyperform. For $p=0$ a hyperform is also called a derivative $r$-form; for $r=0$ a hyperform is a differential $p$-form.

\section{Integration on Superspace and Supermanifolds}

The standard method in "supersymmetric physics" to evaluate integrals of functions on superspace is the following: after expanding a $G^{\infty}$ function $f\left(z^{M}\right)$ from $U \subset M^{\left(D_{0}, D_{1}\right)}$ to $B_{L}$ as

$$
f\left(x^{\mu}, \theta^{\alpha}\right)=f_{0}(x)+f_{\alpha}(x) \theta^{\alpha}+\ldots+f_{T}(x) \theta^{D_{1}} \ldots \theta^{1},
$$


one evaluates the Berezin integral $\not \dot{\beta}$ as

$$
\not{B} f(x, \theta) d^{D_{0}} x d \theta^{1} \ldots d \theta^{D_{1}}=\mathbb{R} f_{T}(x) d^{D_{0}} x,
$$

where the $\mathbb{R}$ integral is meant to be an ordinary Riemann integral. Formally this is achieved by the Berezin integration rules

$$
\not d \theta=0, \quad \oiint \theta d \theta=1 \text {. }
$$

These rules are as they stand void of any measure-theoretic meaning (no integration limits are prescribed). They demand that under a change of variables $(x, \theta) \rightarrow(\bar{x}, \bar{\theta})$ the "volume element" $d^{D_{0}} x d^{D_{1}} \theta$ transforms according to

$$
d^{D_{0}} x d^{D_{1}} \theta=\mathscr{B}(\bar{x}, \bar{\theta}) d^{D_{0}} \bar{x} d^{D_{1}} \bar{\theta},
$$

where $\mathscr{B}$ is the superdeterminant of the matrix

$$
M=\left(\begin{array}{ll}
\frac{\partial x^{\mu}}{\partial \bar{x}^{v}} & \frac{\partial x^{\mu}}{\partial \bar{\theta}^{\beta}} \\
\frac{\partial \theta^{\alpha}}{\partial \bar{x}^{v}} & \frac{\partial \theta^{\alpha}}{\partial \bar{\theta}^{\beta}}
\end{array}\right),
$$

i.e.,

$$
\mathscr{B}=\operatorname{sdet} M=\operatorname{det}\left(\frac{\partial x^{\mu}}{\partial \bar{x}^{\nu}}\right) \operatorname{det}\left(\frac{\partial \bar{\theta}^{\alpha}}{\partial \theta^{\beta}}\right) \text {. }
$$

For $D_{1}=0$ this reduces to $\mathscr{B}=\mathscr{J}=\operatorname{det}\left(\frac{\partial x^{\mu}}{\partial \bar{x}^{v}}\right)$, and for $D_{0}=0$ to $\mathscr{B}=\mathscr{J}^{-1}$ $=\operatorname{det}\left(\frac{\partial \bar{\theta}^{\alpha}}{\partial \theta^{\beta}}\right)$.

We would like to point out that, contrary to common belief, the expression (3.2) is not invariant under a change of variables for arbitrary $f(z)$. This failure is not caused by the Berezin rules (3.3), but by the fact that the $x^{\mu}$ are even Grassmann algebra elements, instead of real variables [13]. The ill-defined recipe (3.2) can however be replaced by a well-defined one $[4,13]$, essentially by treating the integration with respect to the even variables as a contour integration.

For integrals on supermanifolds the procedure will roughly be to define integration in a chart by integration in the model space $B_{L}^{\left(D_{0}, D_{1}\right)}$, and then to patch the results from the different charts together.

To perform the first step, one has to make sure that one integrates objects on the manifold which locally have the same transformation properties as the integrands in the model space. It is well known that for $D$-dimensional $C^{\infty}$ manifolds these objects are $D$-forms. This is also true for $G^{\infty}$ supermanifolds $M^{\left(D_{0}, 0\right)}$. Both the volume element $d^{D_{0}} x$ and the $\left(D_{0}, 0\right)$ hyperform

$$
\Omega_{\left(D_{0}, 0\right)}=\frac{1}{D_{0} !} d x^{\mu_{1}} \wedge \ldots \wedge d x^{\mu_{D_{0}}}\left(_{\mu_{D_{0}} \ldots \mu_{1}} \varepsilon\right)
$$

$\left({ }_{\mu_{D_{0}} \ldots \mu_{1}} \varepsilon\right)$ being the Levi-Civita symbol, transform with the Jacobian determinant: if $\left\{x^{\mu}\right\} \rightarrow\left\{\bar{x}^{\mu}\right\}, \Omega_{\left(D_{0}, 0\right)} \rightarrow \mathscr{J} \bar{\Omega}_{\left(D_{0}, 0\right)}$. This comes about since the (graded antisymmetric) $\wedge$ product is antisymmetric for even differential one-forms $d x^{\mu}$. 
In the other extreme case, i.e. a supermanifold with only odd coordinates, the "volume element" $d^{D_{1}} \theta$ in the model space $B_{L}^{\left(0, D_{1}\right)}$ transforms with $\mathscr{B}=\mathscr{J}^{-1}$ (see (3.4)). This shows that a " $d \theta$ " in $d^{D_{1}} \theta$, despite its appearance, cannot be a differential one-form, and that the volume form cannot be a differential $D_{1}$-form. The object with the appropriate transformation property is the derivative $D_{1}$-form

$$
\Omega_{\left(0, D_{1}\right)}=\frac{1}{D_{1} !} \varepsilon^{\alpha_{1} \ldots \alpha_{D_{1}}}{ }_{\alpha_{1}} \partial \vee \ldots \vee \alpha_{\alpha_{1}} \partial .
$$

This is because the (graded symmetric) $\vee$ product is an antisymmetric product for the odd derivative one-forms ${ }_{\alpha} \partial$, and since ${ }_{\alpha} \partial=\frac{\partial \bar{\theta}^{\beta}}{\partial \theta^{\alpha} \beta} \bar{\partial}\left({ }_{\beta} \bar{\partial}:=\frac{\vec{\partial}}{\partial \bar{\theta}^{\beta}}\right) ;$ therefore $\Omega_{\left(0, D_{1}\right)}$
$=\mathscr{J}^{-1} \bar{\Omega}_{\left(0, D_{1}\right)}$.

The previous cases lead one to suspect that the volume form on $M^{\left(D_{0} . D_{1}\right)}$ is the $\left(D_{0}, D_{1}\right)$ hyperform

$$
\begin{aligned}
\Omega_{\left(D_{0}, D_{1}\right)} & =\Omega_{\left(D_{0}, 0\right)} \otimes \Omega_{\left(0, D_{1}\right)} \\
& =\frac{1}{D_{0} !} \frac{1}{D_{1} !}\left(d x^{\mu_{1}} \wedge \ldots \wedge d x^{\mu_{D_{0}}}{ }_{\mu_{D_{0}} \ldots \mu_{1}} \varepsilon\right) \otimes\left(\varepsilon^{\alpha_{1} \ldots \alpha_{D_{1}}}{ }_{\alpha_{D_{1}}} \partial \wedge \ldots \wedge \alpha_{1} \partial\right) .
\end{aligned}
$$

Under a change of coordinates $(x, \theta) \rightarrow(\bar{x}, \bar{\theta})$,

$$
\begin{gathered}
d x^{\mu}=d \bar{x}^{\nu} \frac{\partial x^{\mu}}{\partial \bar{x}^{\nu}}+d \bar{\theta}^{\alpha} \frac{\partial x^{\mu}}{\partial \bar{\theta}^{\alpha}}, \\
\alpha=\frac{\partial \bar{x}^{\mu}}{\partial \theta^{\alpha}} \bar{\partial}+\frac{\partial \bar{\theta}^{\beta}}{\partial \theta^{\alpha}} \bar{\partial}^{\bar{\partial}} .
\end{gathered}
$$

So one immediately observes that, whereas $\Omega_{\left(D_{0}, D_{1}\right)}$ is a product of even differential forms $d x^{\mu}$ and odd derivative forms ${ }_{\alpha} \partial$, a coordinate transformation leads to terms containing odd differential forms $d \bar{\theta}^{\alpha}$ and even derivative forms ${ }_{\mu} \bar{\partial}$ :

$$
\Omega_{\left(D_{0}, D_{1}\right)}=\mathscr{B} \bar{\Omega}_{\left(D_{0}, D_{1}\right)}+R_{\left(D_{0}, D_{1}\right)},
$$

where $R_{\left(D_{0}, D_{1}\right)}$ contains all unwanted terms with factors $d \bar{\theta}^{\alpha}$ or ${ }_{\mu} \bar{\partial}$.

Berezin [9] proposed to define the integral of a $\left(D_{0}, D_{1}\right)$ hyperform only with respect to the part that transforms correctly (i.e. with $\mathscr{B}$ ). In our notation, if $A$ is a $\left(D_{0}, D_{1}\right)$ hyperform,

$$
A=\frac{1}{D_{0} !} \frac{1}{D_{1} !} d z^{M_{1}} \wedge \ldots \wedge d z^{M_{D_{0}}}\left({ }_{M_{D_{0}} \ldots M_{1}} A^{N_{1} \ldots N_{D_{1}}}\right) \otimes_{N_{D_{1}}} \partial \vee \ldots \vee{ }_{N_{1}} \partial
$$

one may split it as $A=A^{P}+A^{R}$, where $A^{P}$ is proportional to $\Omega_{\left(D_{0}, D_{1}\right)}$, $A^{P}=a(z) \Omega_{\left(D_{0}, D_{1}\right)}$. Berezin proposes

$$
\int A=\int A^{P}=\not \& a(x, \theta) d x^{1} \ldots d x^{D_{0}} d \theta^{1} \ldots d \theta^{D_{1}} .
$$

If we change coordinates $z \rightarrow \bar{z}=h(z)$ and denote $A$ expressed in terms of $\bar{z}$ as $\bar{A}$, then

$$
\int \bar{A}=\int \bar{A}^{P}=\hat{B}\left(a h^{-1}\right) \mathscr{B} d \bar{x}^{1} \ldots d \bar{x}^{D_{0}} d \theta^{1} \ldots d \theta^{D_{1}}
$$


which is consistent with the transformation of the Berezin integral under the same coordinate transformation.

Although Berezin's proposal leads to a consistent definition, one feels unsure about the neglection of the terms $A^{R}$. One would like to have a differentialgeometric, i.e. ultimately a chart independent, understanding of this procedure. This is offered by the observation from (3.8), that all the terms in $R_{\left(D_{0}, D_{1}\right)}$ (see 3.9) contain factors $\frac{\partial x^{\mu}}{\partial \bar{\theta}^{\alpha}}$ and/or $\frac{\partial \bar{x}^{\mu}}{\partial \theta^{\alpha}}$. Therefore $R_{\left(D_{0}, D_{1}\right)}$ would be identically zero, if one were to restrict the coordinate change by requiring $\frac{\partial \bar{x}^{\mu}}{\partial \theta^{\alpha}}=0$. Equally, if $A^{R} \equiv 0$ in one chart, this would imply $\bar{A}^{R} \equiv 0$ in the new chart. Such restricted coordinate transformations "smell like" bundle morphisms in the following sense (the precise notions will be given in the next section; here an informal approach is sufficient): we regard the supermanifold $M^{\left(D_{0}, D_{1}\right)}$ as the total space of a fibre bundle $E=\left(M^{\left(D_{0}, D_{1}\right)}, \pi, B_{0}, F_{1}\right)$ with an (even) base space $B_{0}$, and (odd) fibre $F_{1}$. Natural coordinates in $M^{\left(D_{0}, D_{1}\right)}$ are coordinates inherited from coordinates in $B_{0}$ (via a section) and coordinates in the fibre $F_{1}$, which may be different (i.e. $x$ dependent) for each fibre. A bundle morphism is described locally by a change

$$
\left\{x^{\mu}, \theta^{\alpha}\right\} \rightarrow\left\{\bar{x}^{\mu}(x), \bar{\theta}^{\alpha}(x, \theta)\right\}
$$

from one set of natural coordinates to another. This corresponds to the choice of a different section and a change of basis in the fibres.

If $E$ possesses a connection, the canonical basis in the tangent space to $M^{\left(D_{0}, D_{1}\right)}$ is $\left\{{ }_{\mu} D={ }_{\mu} \partial+{ }_{\mu} \gamma_{\alpha}^{\alpha} \partial,{ }_{\alpha} \partial\right\}$, where $\left\{_{\mu} D\right\}$ is called the horizontal lift of $\left\{_{\mu} \partial\right\}$ into the bundle. $\left\{_{\mu} D\right\}$ and $\left\{{ }_{\alpha} \partial\right\}$ are bases for the horizontal and vertical tangent spaces of $M^{\left(D_{0}, D_{1}\right)}$. Under bundle morphisms (3.12) ${ }_{\mu} \gamma^{\alpha}$ transforms like a connection

$$
{ }_{\mu} \bar{\gamma}^{\alpha}=\frac{\partial x^{\nu}}{\partial \bar{x}^{\mu}} \nu \gamma^{\beta} \frac{\partial \bar{\theta}^{\alpha}}{\partial \theta^{\beta}}+\frac{\partial x^{\nu}}{\partial \bar{x}^{\mu}} \frac{\partial \bar{\theta}^{\alpha}}{\partial x^{\nu}},
$$

such that

$$
{ }_{\mu} D=\frac{\partial \bar{x}^{\nu}}{\partial x^{\mu}}{ }_{\nu} \bar{D}, \quad{ }_{\alpha} \partial=\frac{\partial \bar{\theta}^{\beta}}{\partial \theta^{\alpha}}{ }_{\beta} \bar{\partial} .
$$

The canonical bases (with respect to natural coordinates) in the cotangent spaces to $B_{0}, F_{1}$ and $M^{\left(D_{0}, D_{1}\right)}$ are respectively $\left\{d x^{\mu}\right\},\left\{d \theta^{\alpha}\right\}$ and $\left\{d x^{\mu}, D \theta^{\alpha}=d \theta^{\alpha}\right.$ $\left.-d x^{\mu}{ }_{\mu} \gamma^{\alpha}\right\}$. Under bundle morphisms (3.12) this gives

$$
d x^{\mu}=\frac{\partial x^{\mu}}{\partial \bar{x}^{\nu}} d \bar{x}^{v}, \quad D \theta^{\alpha}=\frac{\partial \theta^{\alpha}}{\partial \bar{\theta}^{\beta}} D \bar{\theta}^{\beta}
$$

The hyperform (3.7) is then the volume form in $M^{\left(D_{0}, D_{1}\right)}$ in the canonical basis. We call it a $\left(D_{0}^{H}, D_{1}^{V}\right)$ hyperform. In later sections it will also be called a $\left(D_{0}, D_{1}\right)$ $P$-hyperform.

We are now in a position to make precise the relationship between the supermanifold integration and the Berezin/Riemann integration:

Definition 1. Let $M^{\left(D_{0}, D_{1}\right)}$ be the total space of a fibre bundle $E=\left(M^{\left(D_{0}, D_{1}\right)}, \pi, B_{0}\right.$, $\left.F_{1}\right)$, and let $p \in M^{\left(D_{0}, D_{1}\right)}$. Let $\left(\pi^{-1}(U), \varphi\right)$ be a chart on the $G^{\infty}$ supermanifold, 
where $U \subset B_{0}$ and $\pi(p) \in U$. Because of the local trivialisation property we take $\pi^{-1}(U) \cong U \times F_{1}$. Let $A$ be a $\left(D_{0}^{H}, D_{1}^{V}\right)$ hyperform with compact support in $\varphi(U)$ $C O$, where $O$ is open in $B_{L, 0}^{D_{0}}$. In natural coordinates $z^{M}=\varphi^{M}(p)$, and with respect to the canonical bases,

$$
A=a(z) \Omega_{\left(D_{0}, D_{1}\right)}=\frac{1}{D_{0} !} \frac{1}{D_{1} !} d x^{\mu_{1}} \wedge \ldots \wedge d x^{\mu_{D_{0}}}{ }_{\mu_{D_{0}} \ldots \mu_{1}} A^{\alpha_{1} \ldots \alpha_{D_{1}}}{ }_{\alpha_{D_{1}}} \partial \vee \ldots \vee_{\alpha_{1}} \partial .
$$

Then

$$
\int_{\pi^{-1}(u)} A:=\underset{O \times B_{L, 1}^{D_{1}}}{\oiint_{1}} a(x, \theta) d x^{1} \ldots d x^{D_{0}} d \theta^{1} \ldots d \theta^{D_{1}} .
$$

We remark that it makes no sense to demand that $A$ has compact support "in the $\theta$ direction," as e.g. for $B_{L}^{(0,1)}$ the only $G^{\infty}$ function of $\theta$ which has compact support is $f(\theta)=0$. By construction the left-hand side of the definition (3.13) transforms with the Berezinian under a change of natural coordinates (3.12). We emphasize that this definition is no less a recipe than the equivalent definition relating integrals of differential forms to Riemann integrals. The recipe consists of replacing the oneforms $d x^{\mu}$ by the integration symbols " $d x^{\mu}$ ", the derivative one-forms ${ }_{\alpha} \partial$ by the integration symbols " $d \theta^{\alpha}$ ", and deleting the $\wedge$ and $\vee$ products. This procedure is justified by the fact that both sides transform identically under the coordinate transformations (3.12).

At first sight the restriction to bundle morphisms, described in natural coordinates by (3.12), seems too strong, as it appears to exclude supersymmetry transformations $\left(x^{\mu} \rightarrow x^{\mu}+\left(\gamma^{\mu}\right)_{\alpha \beta} \theta^{\alpha} \eta^{\beta}, \theta^{\alpha} \rightarrow \theta^{\alpha}+\eta^{\alpha}, \eta^{\alpha}\right.$ constant $)$. However one should observe that in non-natural coordinates $\left(y^{\mu}, \psi^{\alpha}\right)$ a $\left(D_{0}^{H}, D_{1}^{V}\right)$ hyperform is not just a product of $d y^{\mu}$ and $\frac{\vec{\partial}}{\partial \psi^{\alpha}}$ terms. To make this point clear, we demonstrate what happens if one chooses coordinates

$$
y^{\mu}=x^{\mu}+\theta_{\alpha}^{\alpha} \lambda^{\mu}, \quad \psi^{\alpha}=\theta^{\alpha}, \quad \alpha^{\lambda^{\mu} \in B_{L, 1},}
$$

instead of the (natural) coordinates $\left\{x^{\mu}, \theta^{\alpha}\right\}$. One finds

$$
{ }_{\alpha} \partial={ }_{\alpha} \lambda^{\mu} \frac{\partial}{\partial y^{\mu}}+\frac{\vec{\partial}}{\partial \psi^{\alpha}}=:_{\alpha} e, \quad d x^{\mu}=d y^{\mu}-d \psi_{\alpha}^{\alpha} \lambda^{\mu}=: e^{\mu},
$$

and the volume form becomes

$$
\Omega_{\left(D_{0}, D_{1}\right)}=\frac{1}{D_{0} !} \frac{1}{D_{1} !} e^{\mu_{1}} \wedge \ldots \wedge e^{\mu_{D_{0}}}{ }_{\mu_{D_{0}} \ldots \mu_{1}} \varepsilon \otimes \varepsilon^{\alpha_{1} \ldots \alpha_{D_{1}}}{ }_{\alpha_{D_{1}}} e \vee \ldots \vee \alpha_{\alpha_{1}} e
$$

and although containing terms with $\frac{\partial}{\partial y^{\mu}}$ and $d \psi^{\alpha}$, this form is the natural choice with respect to the bundle structure. The integral of a $\left(D_{0}^{H}, D_{1}^{V}\right)$ hyperform $A=a(y, \psi) \Omega_{\left(D_{0}, D_{1}\right)}$ is defined by

$$
\int A=\$ B(y, \psi) d y^{1} \ldots d y^{D_{0}} d \psi^{1} \ldots d^{D_{1}},
$$

i.e. replacing the $e^{\mu}$ and ${ }_{\alpha} e$ on the left-hand side by the symbols $d y^{\mu}$ and $d \psi^{\alpha}$ in the Berezin integral on the right-hand side (and deleting the $\wedge$ and $\vee$ products). Again the definition is independent of the choice of coordinates, provided the 
coordinate change corresponds to a bundle morphism. The restriction $\frac{\partial \bar{x}^{\mu}}{\partial \theta^{\alpha}}=0$ for changes of natural coordinates places a restriction on transformations $\left(y^{\mu}, \psi^{\alpha}\right)$ $\rightarrow\left(\bar{y}^{\mu}, \bar{\psi}^{\alpha}\right)$ of non-natural coordinates: one finds

$$
\bar{y}^{\mu}(y, \psi)=f^{\mu}\left(y^{\nu}-\psi_{\alpha}^{\alpha} \lambda^{\nu}\right)+g^{\alpha}(y, \psi)_{\alpha} \lambda^{\mu}, \quad \bar{\psi}^{\alpha}(y, \psi)=g^{\alpha}(y, \psi)
$$

with $f^{\mu}$ and $g^{\alpha}$ being $G^{\infty}$ functions of the arguments indicated. Furthermore

$$
e^{\mu}=\bar{e}^{\nu}\left(\frac{\partial y^{\mu}}{\partial \bar{y}^{\nu}}-\frac{\partial \psi^{\alpha}}{\partial \bar{y}^{\nu}} \lambda^{\mu}\right), \quad{ }_{\alpha} e=\left(\lambda^{\mu}{ }^{\mu} \frac{\partial \bar{\psi}^{\beta}}{\partial y^{\mu}}+\frac{\partial \bar{\psi}^{\beta}}{\partial \psi^{\alpha}}\right)_{\beta} e,
$$

and hence $\Omega_{\left(D_{0}, D_{1}\right)}$ transforms into $\mathscr{B}(y, \psi) \bar{\Omega}_{\left(D_{0}, D_{1}\right)}$, where $\mathscr{B}(y, \psi)$ is the Berezinian $\operatorname{det}\left(\frac{\partial x^{\mu}}{\partial \bar{x}^{v}}\right) \cdot \operatorname{det}\left(\frac{\partial \bar{\theta}^{\alpha}}{\partial \theta^{\beta}}\right)$ written in $(y, \psi)$ coordinates. As the Berezinians of the other transformations $(y, \psi) \rightarrow(x, \theta)$ and $(\bar{x}, \bar{\theta}) \rightarrow(\bar{y}, \bar{\psi})$ are both 1 , this gives precisely the correct answer, i.e. $\operatorname{det}\left(\frac{\partial y^{\mu}}{\partial \bar{y}^{v}}\right) \cdot \operatorname{det}\left(\frac{\partial \bar{\psi}^{\alpha}}{\partial \psi^{\beta}}\right)$.

This example suggests the possibility of an integration on supermanifolds which have the opposite fibration to $E=\left(M^{\left(D_{0}, D_{1}\right)}, \pi, B_{0}, F_{1}\right)$. By this we mean a fibration characterized by odd coordinates in the base and even coordinates in the fibre. We denote it by $E=\left(M^{\left(D_{0}, D_{1}\right)}, \pi, B_{1}, F_{0}\right)$. Natural coordinates $\left\{\theta^{\alpha}, x^{\mu}\right\}$ on $M^{\left(D_{0}, D_{1}\right)}$ are induced from coordinates on $B_{1}$ and $F_{0}$ respectively, similarly to the previous case. The canonical bases for the tangent spaces of $B_{1}, F_{0}$ and $M^{\left(D_{0}, D_{1}\right)}$ are $\left.\left\{{ }_{\alpha} \partial\right\},{ }_{\mu} \partial\right\}$ and $\left\{{ }_{\alpha} D={ }_{\alpha} \partial+{ }_{\alpha} \Gamma_{\mu}^{\mu} \partial,{ }_{\mu} \partial\right\}$ respectively. The canonical bases for the cotangent spaces are $\left\{d \theta^{\alpha}\right\},\left\{d x^{\mu}\right\}$ and $\left\{d \theta^{\alpha}, D x^{\mu}=d x^{\mu}-d \theta^{\alpha}{ }_{\alpha} \Gamma^{\mu}\right\}$. Bundle morphisms are coordinate transformations $\left\{\theta^{\alpha}, x^{\mu}\right\} \rightarrow\left\{\bar{\theta}^{\alpha}(\theta), \bar{x}^{\mu}(x, \theta)\right\}$ (in natural coordinates). They transform horizontal bases $\left(\left\{_{\alpha} D\right\}\right.$ and $\left.\left\{d \theta^{\alpha}\right\}\right)$ into horizontal ones and vertical bases $\left(\left\{_{\mu} \partial\right\}\right.$ and $\left.\left\{D x^{\mu}\right\}\right)$ into vertical ones. The natural volume form is the $\left(D_{0}^{V}, D_{1}^{H}\right)$ hyperform

$$
\Omega_{\left(D_{0}, D_{1}\right)}=\frac{1}{D_{0} !} \frac{1}{D_{1} !}\left(D x^{\mu_{1}} \wedge \ldots \wedge D x^{\mu_{D_{0}}}{ }_{\mu_{D_{0}} \ldots \mu_{1}} \varepsilon\right) \otimes\left(\varepsilon^{\alpha_{1} \ldots \alpha_{D_{1}}}{ }_{\alpha_{D_{1}}} D \vee \ldots \vee{ }_{\alpha_{1}} D\right)
$$

By construction it transforms with the Berezinian under bundle morphism. This allows us to define the integral of a $\left(D_{0}^{V}, D_{1}^{H}\right)$ hyperform:

Definition 2. Let $M^{\left(D_{0}, D_{1}\right)}$ be the total space of a fibre bundle $E=\left(M^{\left(D_{0}, D_{1}\right)}, \pi, B_{1}\right.$, $\left.F_{0}\right)$, and let $p \in M^{\left(D_{0}, D_{1}\right)}$. Assuming the bundle is trivial, i.e. there is a homeomorphism $h: B_{1} \times F_{0} \rightarrow \pi^{-1}\left(B_{1}\right)$, let $\left(h\left(B_{1} \times U\right), \varphi\right)$ be a chart in $M^{\left(D_{0}, D_{1}\right)}$ with $p \in h\left(B_{1}\right.$ $\times U$ ) and $U$ open in $B_{L, 0}^{D_{0}}$. Let $A$ be a $\left(D_{0}^{V}, D_{1}^{H}\right)$ hyperform with (for all $b \in B_{1}$ ) compact support in $\varphi(h(b \times U)) \subset O$, where $O$ open in $B_{L, 0}^{D_{0}}$. In natural coordinates $z^{M}=\varphi^{M}(p)$, and in the canonical bases, $A$ may be written

$$
A=a(z) \Omega_{\left(D_{0}, D_{1}\right)}=\frac{1}{D_{0} !} \frac{1}{D_{1} !}\left(D x^{\mu_{1}} \wedge \ldots \wedge D x^{\mu_{D_{0}}}\right)_{\mu_{D_{0} \ldots \mu_{1}}} A^{\alpha_{1} \ldots \alpha_{D_{1}}}\left({ }_{\alpha_{D_{1}}} D \vee \ldots \vee_{\alpha_{1}} D\right) .
$$

Then we define

$$
\int_{h\left(B_{1} \times U\right)} A:=\underset{B_{L, 1}^{D} \times O}{\not} a(x, \theta) d x^{1} \ldots d x^{D_{0}} d \theta^{1} \ldots d \theta^{D_{1}} .
$$


We are not quite ready with the complete definition of integration on supermanifolds because in the previous definitions the integration was only defined on appropriate charts of $M^{\left(D_{0}, D_{1}\right)}$. The chart integrations should ultimately be patched together over the manifold. For $C^{\infty}$ manifolds one gets rid of the demand for compact support of a $D$-form in the domain of its chart map by using a partition of unity, which exists if the manifold is paracompact. For both definitions (3.12) and (3.15), the charts for which integration is explained in terms of Berezin/Riemann integration cover the complete odd sector of $M^{\left(D_{0}, D_{1}\right)}$. Hence there is no patching in the odd directions. For the even sector, assuming paracompactness, the patching can again be performed by introducing a partition of unity.

In this section we argued in a local manner. This is sufficient for defining supermanifold integrals in terms of superspace integrals, provided the objects one is dealing with can be given a coordinate independent definition. In addition, knowing the objects which are relevant for integration is not enough, because one ultimately wants to manipulate them without referring to charts. This is possible if one has an exterior calculus for hyperforms. Such a calculus will be presented in Sects. 5 and 6, after we define in a chart independent way horizontal and vertical tensor fields in the next section.

\section{Hyperforms and $\boldsymbol{P}$-Hyperforms}

We showed in the last section that a satisfactorily geometric interpretation of integration on a $G^{\infty}$ supermanifold $M^{\left(D_{0}, D_{1}\right)}$ is possible if one considers the supermanifold (for $D_{0} \neq 0, D_{1} \neq 0$ ) as the total space of a fibre bundle with connection. Two types of fibrations are possible, namely $\left(M^{\left(D_{0}, D_{1}\right)}, \pi, B_{0}, F_{1}\right)$ with even base and odd fibre, and $\left(M^{\left(D_{0}, D_{1}\right)}, \pi, B_{1}, F_{0}\right)$ with odd base and even fibre (even and odd referring to the grading of the coordinates). For application to supersymmetric field theories, the second choice seems to be more appropriate, as we shall explain below. Therefore in the following we shall develop the differential geometry for $\left(M^{\left(D_{0}, D_{1}\right)}, \pi, B_{1}, F_{0}\right)$, and point out at the end the changes that occur for the other fibration.

1. The fibre bundle $E=\left(M^{\left(D_{0}, D_{1}\right)}, \pi, B_{1}, F_{0}\right)$ has as total space $M^{\left(D_{0}, D_{1}\right)}$, a $G^{\infty}$ supermanifold modelled over $B_{L, 1}^{D_{1}} \otimes B_{L, 0}^{D_{0}}$. The projection $\pi$ maps from $M^{\left(D_{0}, D_{1}\right)}$ to $B_{1}$, and we have the local trivializations $\pi^{-1}(U) \cong U \times F_{0}$ for $U$ open in $B_{1}$. The fibre $\pi^{-1}(b)$ is isomorphic to $F_{0}$ for all $b \in B_{1}$.

Locally, as described in the previous section, we have natural coordinates $\left\{z^{N}\right.$ $\left.=\varphi^{N}(m)\right\}=\left\{\theta^{\alpha}, x^{\mu}\right\}$, which are inherited from coordinates in the base and the fibre.

2. We assume that $E$ possesses a connection. Then, for each $m \in E$, there exists a unique split of the tangent space $\underset{(m)}{T} E$ (for convenience we will frequently refer to $E$ when we actually mean the total space $M^{\left(D_{0}, D_{1}\right)}$ of $E$ ):

$$
\underset{(m)}{T} E=\operatorname{hor} \underset{(m)}{T} E \oplus \operatorname{ver} \underset{(m)}{T} E,
$$

such that

$$
\pi_{*}(\operatorname{hor} \underset{(m)}{T} E)=\underset{\pi(m)}{T} B_{1}
$$

where $\pi_{*}: \underset{(m)}{T} E \rightarrow \underset{\pi(m)}{T} B$ is the push-forward induced by the projection $\pi$. 
Locally we choose a basis $\left\{{ }_{M} E\right\}$ in $\underset{(m)}{T} E$, which splits into a basis $\left\{{ }_{\alpha} E=:-E_{\alpha}\right\}$ for hor $\underset{(m)}{T} E$, and $\left\{{ }_{\mu} E=: E_{\mu}\right\}$ for ver $\underset{(m)}{T} E$. In a natural coordinate basis $\left\{{ }_{M} E\right\} \hat{=}\left\{{ }_{\alpha} D\right.$ $\left.={ }_{\alpha} \partial+{ }_{\alpha} \Gamma_{\mu}^{\mu} \partial,{ }_{\mu} \partial\right\}$ where ${ }_{\alpha} \Gamma^{\mu}$ are the components of the connection one-form in $B_{1}$ (see below). These notions of horizontality and verticality extend naturally to (contravariant) vector fields $X \in \mathfrak{X}(E)$ (i.e. the space of sections of the tangent bundle).

3. The action of a (super) vector field $X$ on functions $f \in \mathfrak{F}(U)$ is denoted by $X \cdot f$. The vector fields form a module over the ring of functions with an additional (super) Lie algebra sturcture:

$$
\begin{gathered}
{[,\}: \mathfrak{X}(E) \times \mathfrak{X}(E) \rightarrow \mathfrak{X}(E)} \\
(X, Y) \mapsto[X, Y\}
\end{gathered}
$$

with $[X, Y\} \cdot f=X \cdot(Y \cdot f)-(-1)^{(X)(Y)} Y \cdot(X \cdot f)$. Here and in the following we assume that all tensor fields introduced are pure, i.e. have a definite grading. Due to the split (4.1) of $\underset{(m)}{T E} E$ any vector field splits uniquely as $X=X^{H}+X^{V}$.

Locally, $X=X^{N}{ }_{N} E=E^{N}{ }_{N} X$ (using de Witt conventions [4]). In general we have $\left[{ }_{N} E,{ }_{M} E\right\}={ }_{N M} C^{K}{ }_{K} E$, where ${ }_{N M} C^{K}$ is the object of anholonomicity. In the natural coordinate basis the components of $C$ are zero apart from ${ }_{\alpha \beta} C^{\mu} \hat{=}{ }_{\alpha \beta} \Omega^{\mu}={ }_{\alpha} \partial\left({ }_{\beta} \Gamma^{\mu}\right)$ $+{ }_{\beta} \partial\left({ }_{\alpha} \Gamma^{\mu}\right)$. In this basis $X \cdot f=X^{\alpha}\left({ }_{\alpha} D f\right)+X^{\mu}\left({ }_{\mu} \partial f\right)$.

4. The (super)cotangent space at $m \in E$, denoted $\underset{(m)}{T E}$, is the dual space of $\underset{(m)}{T E}$ i.e. $B_{L}$ linear maps from $\underset{(m)}{T E}$ to $\left.B_{L}: \underset{(m)}{T^{*}} E=L_{B_{L}} \underset{(m)}{T E} ; B_{L}\right)$. The action of an element $\omega$ of $\underset{(m)}{T^{*} E}$ on an element $X$ of $\underset{(m)}{T E}$ is denoted $\langle X \mid \omega\rangle$. (Following the practice in most of the literature we will not distinguish in our notation between elements of

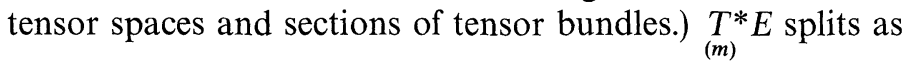

$$
\underset{(m)}{T^{*} E} E=\operatorname{hor} \underset{(m)}{T^{*}} E \oplus \operatorname{ver} \underset{(m)}{T^{*} E}
$$

where $\omega \in$ hor $\underset{(m)}{T^{*} E}$ if $\left\langle X^{V} \mid \omega\right\rangle=0$ for all vectors $X^{V}$ and $\omega \in \operatorname{ver} \underset{(m)}{T^{*} E \text { if }}$ $\left\langle X^{H} \mid \omega\right\rangle=0$ for all vectors $X^{H}$. As before we extend the splitting to covariant vector fields $\omega \in \mathfrak{X}^{*}(E)$.

Locally we choose a basis $\left\{E^{M}:={ }^{M} E\right\}$ in $T_{(m)}^{*} E$, which is dual to the basis $\left\{{ }_{N} E\right\}$ of $\underset{(m)}{T E}:\left\langle{ }_{N} E \mid E^{M}\right\rangle={ }_{N} \delta^{M}$. In a natural coordinate basis we have $\left\{E^{M}\right\} \bumpeq\left\{d \theta^{\alpha}, D x^{\mu}\right.$ $\left.=d x^{\mu}-d \theta_{\alpha}^{\alpha} \Gamma^{\mu}\right\} ; \omega^{H}=d \theta_{\alpha}^{\alpha} \omega, \omega^{V}=D x_{\mu}^{\mu} \omega$. The vertical forms $D x^{\mu}$ define a connection on $E$. Their exterior derivative yields the curvature $d\left(D x^{\mu}\right)=\Omega^{\mu}$ $=\frac{1}{2} d \theta^{\alpha} \wedge d \theta^{\beta}{ }_{\alpha \beta} \Omega^{\mu}$. The pull-back of $D x^{\mu}$ onto $B_{1}$ is $d \theta^{\alpha}{ }_{\alpha} \Gamma^{\mu}$.

5. The super tensor spaces $T_{(m)}^{r}(E)$ at $m \in E$ are defined by the $B_{L}$ multilinear maps:

$$
\left.\underset{(m)}{T_{p}^{r}}(E)=L_{B_{L}} \underset{(m)}{T E} E, \ldots, \underset{(m)}{T} E, \underset{(m)}{T^{*}} E, \ldots, \underset{(m)}{T^{*}} E ; B_{L}\right)=\otimes^{p} \underset{(m)}{T^{*}} E \otimes \otimes_{(m)}^{r} \underset{(m)}{T E}
$$

A tensor field is a section of the tensor bundle $T_{p}^{r}(E)=\bigcup_{m} T_{p}^{r}(E)$. We also define $T_{0}^{0}(E)=\mathfrak{F}(E)$. 
Locally a tensor field $T$ is displayed as

$$
T=E^{N_{1}} \otimes \ldots \otimes E^{N_{p}}\left({ }_{N_{p} \ldots N_{1}} T^{M_{1} \ldots M_{r}}\right)_{M_{r}} E \otimes \ldots \otimes M_{1} E,
$$

where

$$
{ }_{N_{p} \ldots N_{1}} T^{M_{1} \ldots M_{r}}=\left\langle{ }_{N_{p}} E, \ldots, N_{1} E|T| E^{M_{1}}, \ldots, E^{M_{r}}\right\rangle .
$$

6. The hyperform spaces $\Lambda_{p}^{r}(E)$ are defined by the graded multilinear maps

$$
\left.\underset{(m)^{p}}{\Lambda_{p}^{r}}(E)=L_{B_{L}}^{(g)} \underset{(m)}{T} E, \ldots, \underset{(m)}{T} E, T^{*} E, \ldots, \underset{(m)}{T *} E ; B_{L}\right),
$$

which are graded antisymmetric in the first $p$ arguments and graded symmetric in the final $r$ arguments. The elements of $\Lambda_{p}^{r}(E)$ are called $(p, r)$ hyperforms. We also define the direct sum of all $(p, r)$ hyperform spaces $\Lambda(E):=\bigoplus_{p, r} \Lambda_{p}^{r}(E)$, and the spaces $\operatorname{dif} \Lambda(E):=\bigoplus_{p} \Lambda_{p}^{0}(E)$ of differential forms and $\operatorname{der} \Lambda(E):=\bigoplus_{r} \Lambda_{0}^{r}(E)$ of derivative forms.

Locally an element $\varphi$ of $\Lambda_{p}^{r}(E)$ is expressed in terms of the basis

$$
\begin{aligned}
E^{N_{1} \ldots N_{p}} \otimes_{M_{r} \ldots M_{1}} E & =\left(E^{N_{1}} \wedge \ldots \wedge E^{N_{p}}\right) \otimes\left({ }_{M_{r}} E \vee \ldots \vee{ }_{M_{1}} E\right) \\
: & =p ! r ! E^{\left[N_{1}\right.} \otimes \ldots \otimes E^{\left.N_{p}\right)} \otimes_{\left(M_{r}\right.} E \otimes \ldots \otimes_{\left.M_{1}\right]} E,
\end{aligned}
$$

as

$$
\varphi=\frac{1}{p !} \frac{1}{r !} E^{N_{1} \ldots N_{p}} \otimes\left({ }_{N_{p} \ldots N_{1}} \varphi^{M_{r} \ldots M_{1}}\right)_{M_{1} \ldots M_{r}} E .
$$

7. Finally we define the $P$-hyperform spaces $\Omega_{p}^{r}(E) \subset \Lambda_{p}^{r}(E)$ by means of a projection

$$
P: \Lambda_{p}^{r}(E) \rightarrow \Omega_{p}^{r}(E), \quad \varphi \mapsto P \varphi=\varphi^{P},
$$

where

$$
\left\langle X_{1}, \ldots, X_{p}\left|\varphi^{P}\right| \omega_{1}, \ldots, \omega_{r}\right\rangle:=\left\langle X_{1}^{V}, \ldots, X_{p}^{V}|\varphi| \omega_{1}^{H}, \ldots, \omega_{r}^{H}\right\rangle
$$

for all $X_{i} \in \mathfrak{X}(E)$ and $\omega_{i} \in \mathfrak{X}^{*}(E)$. Elements of $\Omega_{p}^{r}(E)$ are called $(p, r)^{P}$ hyperforms or $\left(p^{V}, r^{H}\right)$ hyperforms, as in the differential (derivative) form part the horizontal (vertical) pieces are projected away. For functions $f^{P}=f$, for contravariant vectors $X^{P}=X^{H}$ and for covariant vectors $\omega^{P}=\omega^{V}$. If $\varphi$ and $\bar{\varphi}$ are elements of $\Lambda(E)$, then $P(\varphi \otimes \bar{\varphi})=\varphi^{P} \otimes \bar{\varphi}^{P}$. Analogously to the definition in point 6, we define the spaces $\Omega(E), \operatorname{dif} \Omega(E), \operatorname{der} \Omega(E)$, where the summations now only run over $r \leqq D_{1}$ and $p \leqq D_{0}$.

Locally we have for $\varphi$ given by (4.5):

$$
P \varphi=\varphi^{P}=\frac{1}{p !} \frac{1}{r !} E^{\mu_{1} \ldots \mu_{p}} \otimes\left({ }_{\mu_{p} \ldots \mu_{1}} \varphi^{\alpha_{2} \ldots \alpha_{1}}\right)_{\alpha_{1} \ldots \alpha_{r}} E .
$$

Observe that ${ }_{\mu_{p} \ldots \mu_{1}} \varphi^{\alpha_{r} \ldots \alpha_{1}}$ is completely antisymmetric in both its upper and lower indices. 


\section{Exterior Calculus of Hyperforms}

1. In the spaces of differential and derivative forms, one has an exterior product $(\wedge$ and $\vee$ respectively). We wish to extend this to an exterior product $x$ in $\Lambda(E)$ :

$$
x: \Lambda_{p}^{r}(E) \times \Lambda_{q}^{s}(E) \rightarrow \Lambda_{p+q}^{r+s}(E), \quad(\varphi, \bar{\varphi}) \mapsto \varphi \times \bar{\varphi} .
$$

The product $x$ is defined by its action on the basis elements:

$$
\begin{gathered}
E^{N} \times E^{M}:=E^{N} \wedge E^{M}, \\
{ }_{N} E \aleph_{M} E:={ }_{N} E \vee_{M} E, \\
E^{N} \aleph_{M} E:=E^{N} \otimes_{M} E, \\
{ }_{M} E \rightsquigarrow E^{N}:=(-1)^{(N)(M)} E^{N} \otimes_{M} E .
\end{gathered}
$$

For $\varphi \in \Lambda_{p}^{r}(E), \bar{\varphi} \in \Lambda_{q}^{s}(E)$ we have the symmetry property,

$$
\bar{\varphi} \times\left(\varphi=(-1)^{p q+(\varphi)(\bar{\varphi})} \varphi \rightsquigarrow \bar{\varphi} .\right.
$$

2. A map $\delta: \Lambda_{p}^{r}(E) \rightarrow \Lambda_{p+\pi}^{r}(E)$ defined for all $r, p \geqq 0$ is called a derivation of type $(\pi,(\delta))$, where $(\delta):=(\delta \varphi)-(\varphi)$, if the following properties are satisfied

$$
\begin{gathered}
\delta\left(\Lambda_{p}^{r}(E)\right)=0 \quad \text { for } \quad p+\pi<0, \\
\delta(\varphi \lambda+\bar{\varphi} \bar{\lambda})=(\delta \varphi) \lambda+(\delta \bar{\varphi}) \bar{\lambda} ; \quad \lambda, \bar{\lambda} \in B_{L}, \quad \text { (“B } B_{L} \text {-linearity”), } \\
\delta(\varphi \times \bar{\varphi})=(\delta \varphi) \rightsquigarrow \bar{\varphi}+(-1)^{(\delta, \varphi)} \varphi \rightsquigarrow(\delta \bar{\varphi}), \quad(\delta, \varphi):=(\delta)(\varphi)+\pi p .
\end{gathered}
$$

If $\delta_{i}$ are derivations of type $\left(\pi_{i},\left(\delta_{i}\right)\right)$, the supercommutator

$$
\left[\delta_{i}, \delta_{j}\right\}:=\delta_{i} \delta_{j}-(-1)^{\left(\delta_{i}, \delta_{j}\right)} \delta_{j} \delta_{i}, \quad\left(\delta_{i}, \delta_{j}\right):=\left(\delta_{i}\right)\left(\delta_{j}\right)+\pi_{i} \pi_{j}
$$

is a derivation of type $\left(\pi_{i}+\pi_{j},\left(\delta_{i}\right)+\left(\delta_{j}\right)\right)$. Of course the supercommutator may just be the trivial derivation 0 .

3. Since below we shall arrive at an exterior calculus on $\Lambda(E)$ partly by using the Cartan calculus on dif $\Lambda(E)$, we state here the rules for the latter theory (see e.g. [4]): the exterior derivative $d$ and the contraction $i_{X}$ with respect to a vector field $X$ are derivations on $\operatorname{dif} \Lambda(E)$ of type $(1,0)$ and $(-1,(X))$ respectively, with

$$
\left[i_{X}, i_{Y}\right\}=0, \quad\left[d, i_{X}\right\}=\mathfrak{I}_{X},
$$

where $\mathfrak{L}_{X}$ is the Lie derivative with respect to the vector field $X$. The supercommutators involving $\mathfrak{S}_{X}$ are

$$
\left[\mathfrak{Q}_{X}, d\right\}=0, \quad\left[\mathfrak{L}_{X}, i_{Y}\right\}=i_{[X, Y\}}, \quad\left[\mathfrak{L}_{X}, \mathfrak{L}_{Y}\right\}=\mathfrak{L}_{[X, Y\}} .
$$

4. We extend the Cartan calculus from $\operatorname{dif} \Lambda(E)$ to $\Lambda(E)$ by considering a $(p, r)$ hyperform as a vector-valued differential form:

$$
\begin{aligned}
\varphi & =\frac{1}{r !} \varphi_{(p)}^{M_{1} \ldots M_{r}}{ }_{M_{r} \ldots M_{1}} E, \\
\varphi_{(p)}^{M_{1} \ldots M_{r}} & =\frac{1}{p !} E^{N_{1} \ldots N_{p}}\left({ }_{N_{p} \ldots N_{1}} \varphi^{M_{1} \ldots M_{r}}\right) .
\end{aligned}
$$


So $\varphi_{(p)}^{M_{1} \ldots M_{r}}$ is a differential $p$-form with values in $\Lambda_{0}^{r}(E)$. The derivations introduced below only "see" the differential form part of a hyperform:

$$
\delta \varphi=\frac{1}{r !} \delta\left(\varphi_{(p)}^{M_{1} \ldots M_{r}}\right)_{M_{r} \ldots M_{1}} E
$$

5. The exterior derivative on $\Lambda(E)$ is defined as a covariant derivative on vector-valued forms. It is obtained in the following way: a covariant derivate $\nabla$ on tensor fields is a $B_{L}$ linear map:

$$
\nabla: T_{p}^{r}(E) \rightarrow T_{p+1}^{r}(E)
$$

with

(i) $\nabla f=d f$ for $f \in \mathfrak{F}(E)$,

(ii) $\nabla\left(T_{1} \otimes T_{2}\right)=\nabla T_{1} \otimes T_{2}+T_{1} \otimes \nabla T_{2}$,

(iii) $\langle Y \mid \nabla\langle X \mid \omega\rangle\rangle=\langle Y|\nabla X| \omega\rangle+\langle Y, X| \nabla \omega)$ for $X, Y \in \mathfrak{X}(E), \omega \in \mathfrak{X}^{*}(E)$.

By these requirements $\nabla$ is completely determined on any tensor field by specifying its action on a contravariant base vector ${ }_{N} E$ :

$$
\nabla_{N} E={ }_{N} \omega^{M} \otimes_{M} E
$$

where $\omega$ is the connection one-form (linear connection in $E$ ). The action of $\nabla$ on a hyperform $\varphi$ can be expressed as an action $\tilde{\nabla}$ on the components of $\varphi$ :

$$
\nabla \varphi=\frac{1}{p ! r !} E^{N_{1} \ldots N_{p}} \otimes \tilde{V}\left({ }_{N_{p} \ldots N_{1}} \varphi^{M_{1} \ldots M_{r}}\right) \otimes_{M_{r} \ldots M_{1}} E .
$$

The resulting object is however in general not a hyperform. Therefore we define the exterior covariant derivative $\mathrm{dl}$ on a hyperform as

$$
\begin{gathered}
\mathrm{dl} \varphi=\frac{1}{r !}\left(\mathrm{dl} \varphi_{(p)}^{M_{1} \ldots M_{r}}\right)_{M_{r} \ldots M_{1}} E \\
\mathrm{dl} \varphi_{(p)}^{M_{1} \ldots M_{r}}:=d \varphi_{(p)}^{M_{1} \ldots M_{r}}+r(-1)^{p} \varphi_{(p)}^{\left[M_{1} \ldots M_{r-1} M^{M}\right.} \wedge_{M} \omega^{\left.M_{r}\right)},
\end{gathered}
$$

and get as special cases $\mathrm{dl} \varphi_{(p)}=d \varphi_{(p)}$ and $\mathrm{dl} \varphi_{(0)}^{M_{1} \ldots M_{r}}=\tilde{\nabla} \varphi_{(0)}^{M_{1} \ldots M_{r}}$. dl is a derivation on $\Lambda(E)$ of type $(1,0)$.

6. The contraction of a hyperform with respect to a contravariant vector field $X$ is a map

$$
i_{X}: \mathfrak{X}(E) \times \Lambda_{p}^{r}(E) \rightarrow \Lambda_{p-1}^{r}(E)
$$

with

(i) for fixed $X, i_{X}$ is a derivation of type $(-1,(X))$,

(ii) $\left\langle Y_{1} \ldots Y_{p-1}\left|i_{X} \varphi\right| \omega_{1} \ldots \omega_{r}\right\rangle:=\left\langle Y_{1} \ldots Y_{p-1} X|\varphi| \omega_{1} \ldots \omega_{r}\right\rangle$.

7. It turns out to be convenient to introduce the following notation: let ${ }_{N} W^{M}$ be a matrix of differential $q$-forms. Then for a $(p, r)$ hyperform we define

$$
W \circ \varphi:=(-1)^{p q+(\varphi)(W)} r \varphi_{(p)}^{\left[M_{1} \ldots M_{r-1} M\right.} \wedge_{M} W^{\left.M_{r}\right)}{ }_{M_{r} \ldots M_{1}} E .
$$


Thus we can express $(5.8)$ compactly as $\mathrm{dl}=d+\omega \circ$. It is straightforward to prove the following statements:

(i) If $\delta$ is a derivation in $\Lambda(E)$ of type $(\pi,(\delta))$, then

$$
\delta(W \circ \varphi)=\delta W \circ \varphi+(-1)^{\pi q+(\delta)(W)} W \circ \delta \varphi .
$$

(ii) If $W_{i}$ are matrices of differential $q_{i}$ forms, then

$$
W_{1} \circ\left(W_{2} \circ \varphi\right)=(-1)^{q_{1} q_{2}+\left(W_{1}\right)\left(W_{2}\right)}\left(W_{2} \wedge W_{1}\right) \circ \varphi .
$$

8. The supercommutators of $\mathrm{dl}$ and $i_{X}$ are found to be

$$
[\mathrm{dl}, \mathrm{dl}\}=2(\Omega \circ), \quad\left[\mathrm{d}, i_{X}\right\}=\mathfrak{l}_{X}, \quad\left[i_{X}, i_{Y}\right\}=0,
$$

where $\Omega$ is the curvature

$$
{ }_{N} \Omega^{M}:=d_{N} \omega^{M}-{ }_{N} \omega^{K} \wedge{ }_{K} \omega^{M},
$$

and the covariant Lie derivative $I_{X}$ can be expressed in terms of the Lie derivative $\mathfrak{Q}_{X}$ as

$$
\mathfrak{l}_{X}=\mathfrak{I}_{X}+\left(i_{X} \omega\right) \circ .
$$

Here we take $\mathfrak{Q}_{X}$ to act only on the differential form part of a hyperform. $(\Omega \circ)$ and $\mathfrak{I}_{X}$ are derivations of type $(2,0)$ and $(0,(X))$ respectively.

9. By forming supercommutators with $\mathfrak{I}_{X}$ and $(\Omega \circ)$, one finds

$$
\begin{gathered}
{\left[\mathfrak{l}_{X}, \mathrm{dl}\right\}=\left(i_{X} \Omega\right) \circ,} \\
{\left[\mathfrak{l}_{X}, i_{Y}\right\}=i_{[X, Y\}},} \\
{\left[\mathfrak{l}_{X}, \mathfrak{l}_{Y}\right\}=\mathfrak{l}_{[X, Y\}}-\left(i_{X} i_{Y} \Omega\right) \circ,} \\
{\left[i_{X}, \Omega \circ\right\}=\left(i_{X} \Omega\right) \circ,} \\
{\left[\mathfrak{l}_{X}, \Omega \circ\right\}=\left(\mathfrak{l}_{X} \Omega\right) \circ=\left(\mathrm{dl} i_{X} \Omega\right) \circ .}
\end{gathered}
$$

Thus further derivations appear on the right-hand side of the supercommutators. If we denote by $\delta_{i}$ any of the derivations from the set $\left\{\mathrm{dl}, i_{X}, \mathrm{I}_{X}\right\}$, the chains $\left(\delta_{i} \Omega\right) \circ$, $\left(\delta_{i} \delta_{j} \Omega\right) \circ$, etc. are also derivations. Let us denote these collectively by $\left(\Omega_{I} \circ\right)$. For supercommutators involving $\left(\Omega_{I} \circ\right)$, we have

$$
\begin{gathered}
{\left[\delta_{i}, \Omega_{I} \circ\right\}=\left(\delta_{i} \Omega_{I}\right) \circ,} \\
{\left[\Omega_{I} \circ, \Omega_{J} \circ\right\}=(-1)^{q_{I} q_{J}+\left(\Omega_{I}\right)\left(\Omega_{J}\right)}\left(\Omega_{J} \wedge \Omega_{I}\right) \circ-\left(\Omega_{I} \wedge \Omega_{J}\right) \circ .}
\end{gathered}
$$

\section{Exterior Calculus of $\boldsymbol{P}$-Hyperforms}

1. We define an exterior product $\diamond=P x$,

$$
\diamond: \Lambda_{p}^{r}(E) \times \Lambda_{q}^{s}(E) \rightarrow \Omega_{p+q}^{r+s}(E), \quad(\varphi, \bar{\varphi}) \mapsto \varphi \diamond \bar{\varphi}:=(\varphi \times \bar{\varphi})^{P} .
$$

Since $(\varphi \times \bar{\varphi})^{P}=\varphi^{P} \times \bar{\varphi}^{P}=\varphi^{P} \diamond \bar{\varphi}^{P}$, one has $[P, \times]=P \times x-\times P=0$. Therefore for $P$-hyperforms $\diamond$ and $x$ can be identified.

2. A derivation $\Delta$ of type $(\pi,(\Delta))$ on $\Omega(E)$ is a $B_{L}$ linear map

$$
\Delta: \Omega_{p}^{r}(E) \rightarrow \Omega_{p+\pi}^{r}(E)
$$


with

$$
\Delta(\phi \diamond \bar{\phi})=(\Delta \phi) \diamond \bar{\phi}+(-1)^{(\Delta, \phi)} \phi \diamond(\Delta \bar{\phi}),
$$

and obeying similar postulates to (5.3).

3. If $\delta$ is a derivation in $\Lambda(E)$, it is in general not a derivation in $\Omega(E)$ :

$$
\begin{gathered}
\delta: \Omega_{p}^{r}(E) \rightarrow \Lambda_{p+\pi}^{r}(E), \\
\delta(\phi \diamond \bar{\phi})=(\delta \phi) \diamond \bar{\phi}+(-1)^{(\delta, \phi)} \phi \diamond(\delta \bar{\phi})+R \delta(\phi \diamond \bar{\phi}),
\end{gathered}
$$

where we introduced $R=\mathrm{id}_{A(E)}-P$. As a consequence one can state: if $\delta$ is a derivation of type $(\pi,(\delta))$ in $\Lambda(E)$, then $P \delta$ is a derivation of the same type in $\Omega(E)$. $P \delta$ is in general not a derivation in $\Lambda(E)$ :

$$
\begin{gathered}
P \delta: \Lambda_{p}^{r}(E) \rightarrow \Omega_{p+\pi}^{r}(E), \\
P \delta(\varphi \rightsquigarrow \bar{\varphi})=(P \delta \varphi) \diamond \bar{\varphi}+(-1)^{(\delta, \varphi)} \varphi \diamond(P \delta \bar{\varphi}) \\
=(\delta \varphi) \diamond \bar{\varphi}+(-1)^{(\delta, \varphi)} \varphi \diamond(\delta \bar{\varphi}) \\
\neq(P \delta \varphi) \rightsquigarrow \bar{\varphi}+(-1)^{(\delta, \varphi)} \varphi \rightsquigarrow(P \delta \bar{\varphi}) .
\end{gathered}
$$

4. Let $\delta_{i}$ be derivations of type $\left(\pi_{i},\left(\delta_{i}\right)\right)$ in $\Lambda(E)$. Call $\Delta_{i}=P \delta_{i}$ the corresponding derivations in $\Omega(E)$. By applying $P$ to the supercommutator $\left[\delta_{i}, \delta_{j}\right\}$, one obtains

$$
\Delta_{(i, j)}=P\left[\delta_{i}, \delta_{j}\right\}=\Delta_{i} \delta_{j}-(-1)^{\left(\delta_{i}, \delta_{j}\right)} \Delta_{j} \delta_{i} .
$$

By writing $\delta_{j}=\Delta_{j}+R \delta_{j}$, one gets

$$
\left[\Delta_{i}, \Delta_{j}\right\}=\Delta_{(i, j)}-\left(\psi_{i j}-(-1)^{\left(\delta_{i}, \delta_{j}\right)} \psi_{j i}\right),
$$

where $\psi_{i j}:=\Delta_{i} R \delta_{j}$. One can show that $\psi_{i j}$ is itself a derivation in $\Omega(E)$.

5. We introduced the derivations $\mathrm{dl}$ and $i_{X}$ in $\Lambda(E)$. By projection we get the derivations $D:=P \mathrm{dl}$ and $I_{X}:=P i_{X}$ in $\Omega(E)$. They satisfy the following two special properties:

$$
\begin{gathered}
D P=D, \\
I_{X} P=i_{X} P .
\end{gathered}
$$

We show (i) just for $X \in \mathfrak{X}(E)$ and $\omega \in \mathfrak{X}^{*}(E)$. By the derivation property of $D$, the proof can be extended to arbitrary hyperforms. Firstly $\mathrm{d} X=\left(\mathrm{dl} X^{N}\right)_{N} E$, hence

Secondly we have

$$
D X=\left(\mathrm{d} l X^{\alpha}\right)_{\alpha}^{P} E=D X^{P} .
$$

$$
\begin{aligned}
& \mathrm{dl} \omega=d \omega=d E^{N}{ }_{N} \omega-E^{N} \wedge d_{N} \omega, \\
& D \omega=\left(d E^{N}\right)^{P}{ }_{N} \omega-E^{\mu} \wedge\left(d_{\mu} \omega\right)^{P}, \\
& D \omega^{P}=\left(d E^{\mu}\right)^{P}{ }_{\mu} \omega-E^{\mu} \wedge\left(d_{\mu} \omega\right)^{P},
\end{aligned}
$$

and the result follows from

$$
\begin{aligned}
& d E^{N}=\frac{1}{2} E^{K} \wedge E^{L}{ }_{K L} C^{N}, \\
& \left(d E^{N}\right)^{P}=\frac{1}{2} E^{\mu} \wedge E^{v}{ }_{v \mu} C^{N},
\end{aligned}
$$

when we recall that ${ }_{v \mu} C^{\alpha}=0\left(\right.$ since $\left.\left[X^{V}, Y^{V}\right\}^{H}=0\right)$. 
It is sufficient to show (ii) for differential forms $\omega$. From the definition we have

$$
\begin{aligned}
&\left\langle Y_{1} \ldots Y_{p-1} \mid i_{X} \omega^{P}\right\rangle=\left\langle Y_{1} \ldots Y_{p-1} X \mid \omega^{P}\right\rangle=\left\langle Y_{1}^{P} \ldots Y_{p-1}^{P} X^{P} \mid \omega^{P}\right\rangle \\
&\left\langle Y_{1} \ldots Y_{p-1} \mid P i_{X} \omega^{P}\right\rangle=\left\langle Y_{1}^{P} \ldots Y_{p-1}^{P} \mid i_{X} \omega^{P}\right\rangle \\
&=\left\langle Y_{1}^{P} \ldots Y_{p-1}^{P} X \mid \omega^{P}\right\rangle \\
&=\left\langle Y_{1}^{P} \ldots Y_{p-1}^{P} X^{P} \mid \omega^{P}\right\rangle .
\end{aligned}
$$

This demonstrates that on $\Omega(E)$ the derivation $I_{X}$ is identical with $i_{X}$, and that $i_{X^{H}}=0$ on $\Omega(E)$. A consequence of (6.6) and (6.7) is that some of the derivations $\psi_{i j}$ defined in (6.5) are trivial:

$$
\begin{gathered}
D R \delta_{i} \equiv 0 \\
\delta_{i} R i_{X} \equiv 0 \text { on } \Omega(E) .
\end{gathered}
$$

6. The rules stated in Sect. 5 allow one to obtain the supercommutators of derivations in $\Omega(E)$. We only list those coming from $D$ and $I_{X}$ :

$$
\begin{gathered}
{[D, D\}=2(\Omega \circ)^{P},} \\
{\left[D, I_{X}\right\}=P \mathfrak{I}_{X}-I_{X} R \mathrm{~d} l,} \\
{\left[I_{X}, I_{Y}\right\}=0 .}
\end{gathered}
$$

7. We assume that the supermanifold possesses a Riemannian (super)metric $g \in T_{0}^{2}(E)$ satisfying:

(i) $\langle X, Y \mid g\rangle=(-1)^{(X)(Y)}\langle Y, X \mid g\rangle$.

(ii) $g$ is non-degenerate, i.e. if (for all $Y$ ) $\langle X, Y \mid g\rangle=0$, then $X=0$.

The signature of $g$ denoted $\operatorname{sign}(g)$ is the dimension of the maximal subspace of $\underset{(m)}{T}(E)$ for which $\langle X, X \mid g\rangle<0$ (for all $m$ ).

Locally

$$
\begin{gathered}
g=E^{M} \otimes E^{N}{ }_{N M} g=: E^{M} \otimes{ }_{M} g_{N}{ }^{N} E, \\
{ }_{N} g_{M}=(-1)^{(N)(M)}{ }_{M} g_{N}, \\
|g|:=\left|\operatorname{sdet}\left({ }_{N} g_{M}\right)\right| \neq 0 .
\end{gathered}
$$

Under a change of basis

$$
E^{M}=\bar{E}^{K}{ }_{K} A^{M}=\left({ }^{M} A_{K}\right)^{T K} \bar{E},
$$

where

$$
\left({ }^{M} A_{K}\right)^{T}=(-1)^{K(K+M)}{ }_{K} A^{M}
$$

is the supertranspose of $A, g$ changes according to

$$
g=\bar{E}^{K} \otimes_{K} A^{M}{ }_{M} g_{N}\left({ }^{N} A_{L}\right)^{T} \otimes{ }^{L} \bar{E}=\bar{E}^{K} \otimes_{K} \bar{g}_{L}{ }^{L} \bar{E},
$$

such that

$$
|\bar{g}|=|g|\left(\operatorname{sdet}\left({ }_{N} A^{M}\right)\right)^{2} .
$$

8. One can define a Hodge duality operation $*$ in $\Omega(E)$,

$$
*: \Omega_{p}^{r}(E) \rightarrow \Omega_{D_{0}-p}^{D_{1}-r}(E), \quad \phi \mapsto * \phi .
$$


Locally for $\phi$ given by

$$
\phi=\frac{1}{r !} \frac{1}{p !} E^{\mu_{1} \ldots \mu_{p}}\left({ }_{\mu_{p} \ldots \mu_{1}} \phi^{\alpha_{1} \ldots \alpha_{r}}\right) \diamond_{\alpha_{r} \ldots \alpha_{1}} E
$$

we have

$$
* \phi=\frac{1}{\left(D_{0}-p\right) !} \frac{1}{\left(D_{1}-r\right) !} E^{\mu_{p+1} \ldots \mu_{D_{0}}}\left({ }_{\mu_{D_{0} \ldots \mu_{p}+1}} * \phi^{\alpha_{r+1} \ldots \alpha_{D_{1}}}\right) \diamond_{\alpha_{D_{1} \ldots \alpha_{r+1}}} E
$$

where

$$
\begin{gathered}
\mu_{D_{0} \ldots \mu_{p+1}} * \phi^{\alpha_{r+1} \ldots \alpha_{D_{1}}}:=\frac{|g|^{1 / 2}}{p ! r !}{ }_{\mu_{D_{0}} \ldots \mu_{1}} \varepsilon^{\mu_{1}} g^{v_{1}} \ldots{ }^{\mu_{p}} g^{v_{p}} \\
\diamond\left({ }_{v_{p} \ldots v_{1}} \phi^{\beta_{1} \ldots \beta_{r}}\right)_{\beta_{r}} g_{\alpha_{r} \ldots \beta_{1}} g_{\alpha_{1}} \varepsilon^{\alpha_{1} \ldots \alpha_{D_{1}}},
\end{gathered}
$$

and ${ }^{N} g^{M}$ with upper indices denotes the inverse of ${ }_{N} g_{M}$. From this one can show

$$
\begin{gathered}
* * \phi=(-1)^{\operatorname{sign}(g)}(-1)^{p\left(D_{0}-p\right)}(-1)^{r\left(D_{1}-r\right)} \phi, \\
\phi \diamond * \phi=\frac{1}{p !} \frac{1}{r !}{ }^{\mu_{1} \ldots \mu_{p}} \phi^{\alpha_{1} \ldots \alpha_{r} \mu_{1} \ldots \mu_{p}} \phi_{\alpha_{1} \ldots \alpha_{r}} \Omega,
\end{gathered}
$$

where indices are raised and lowered the metric and its inverse. Furthermore $\Omega=* 1$ is the canonical volume form

$$
\Omega=\sqrt{|g|} \frac{1}{D_{0} !} \frac{1}{D_{1} !} E^{\mu_{1} \ldots \mu_{D_{0}}}{ }_{\mu_{D_{0}} \ldots \mu_{1}} \varepsilon \otimes \varepsilon^{\alpha_{1} \ldots \alpha_{D_{1}}}{ }_{{ }_{D_{1}} \ldots \alpha_{1}} E .
$$

\section{Conclusions}

We have shown that the ad hoc prescription for integration on supermanifolds proposed by Berezin can be justified by postulating that the supermanifold is the total space of a fibre bundle with connection. In the fibration $E=\left(M^{\left(D_{0}, D_{1}\right)}, \pi, B_{1}\right.$, $\left.F_{0}\right)$ the volume hyperform consists of pieces "living in" the horizontal tangent space and the vertical cotangent space of the bundle. The transition from a supermanifold integral to a superspace (Riemann/Berezin) integral only makes sense in canonical bases in the horizontal and vertical tangent and cotangent spaces. Under coordinate transformations which are natural with respect to the bundle structure (i.e. bundle morphisms) the volume hyperform transforms with the Berezinian. Under arbitrary coordinate transformations the invariant notions of horizontality and verticality preserve the right transformation properties.

We extended the Cartan calculus for differential forms to hyperforms by considering these as vector-valued differential forms. This gave rise to a covariant exterior derivative dl. Here we do not agree with Rogers, who in [11] essentially sets $d\left({ }_{N} \partial\right)=0$. As a consequence one would get for a vector field $d\left(X^{M}{ }_{M} \partial\right)$ $=d X^{M} \otimes_{M} \partial=d z^{N}\left({ }_{N} \partial X^{M}\right) \otimes_{M} \partial$. However ${ }_{N} \partial X^{M}$ is not a tensor. The only reasonable thing one can do is to work with covariant derivatives. We described the chain of derivations which arise by forming supercommutators of earlier derivations. Finally, we obtained the exterior calculus for $P$-hyperforms by making use of the 
projection $P$. To our own suprise, this procedure yields derivations not present before the projection. They may not be of much importance for "practical" calculations, but we find them rather intriguing from a structural point of view. At present we are investigating whether one can make general statements about the supercommutators of the additional derivations on $\Omega(E)$. We also plan to enrich the exterior calculus with relations involving the Hodge duality operation.

In the Cartan calculus on differential forms, the Lie derivative $\mathfrak{L}_{X}$ is a derivation obtainable from $d$ and $i_{X}\left(\mathfrak{Q}_{X}=i_{X} d+d i_{X}\right)$. On hyperforms this corresponds to $\mathfrak{l}_{X}=i_{X} \mathrm{dl}+\mathrm{dl} i_{X}$. Like $\mathrm{dl}$ and $i_{X}, \mathfrak{l}_{X}$ acts only on the "differential form part" of a hyperform. For instance, for a $(1,1)$ hyperform:

$$
\mathfrak{l}_{X}\left(\varphi^{N}{ }_{N} E\right)=\left(\mathfrak{l}_{X} \varphi^{N}\right)_{N} E=\left(\mathfrak{I}_{X} \varphi^{N}-\varphi^{M} \wedge i_{X M} \omega^{N}\right)_{N} E .
$$

However $\mathfrak{L}_{X}$ is itself a respectable derivation on hyperforms (acting on both parts) and could be added from the beginning to $\mathrm{dl}$ and $i_{X}$. This could be of use since $\mathfrak{Q}_{X}$ is related to diffeomorphisms and $P \mathfrak{L}_{X}$ to bundle morphisms.

One could also introduce into the exterior calculus a contraction $i_{\omega}$ of a hyperform with a one-form $\omega$ (now only acting on the "derivative form part"). By generalizing the concept of a derivation $\delta$ to maps $\delta: \Lambda_{p}^{r}(E) \rightarrow \Lambda_{p+\pi}^{r+\varrho}(E), i_{\omega}$ would be a derivation with $\pi=0, \varrho=-1$.

We would like to remark that our hyperforms and $P$-hyperforms bear some resemblance to constructs used in gauge theories. There one is also dealing with mixed tensors. A Lie algebra-value $p$-form $A(A \in \Lambda(M, \mathscr{G}) ; \mathscr{G}$ the Lie algebra of the gauge group $G$ ) is given locally by

$$
A=\frac{1}{p !} A_{\mu_{1} \ldots \mu_{p}}^{i} d x^{\mu_{1}} \wedge \ldots d x^{\mu_{p}} \otimes \xi_{i} .
$$

Here the $\xi_{i}$ are the Lie algebra generators, which we may regard as spanning the vertical part of the tangent space of a principal bundle. One can think of $A$ as a $(p, 1)$ hyperform. The exterior product $\wedge$ for vector-valued forms $(A \wedge B$ $\left.=A^{i} \otimes \xi_{i} \wedge B^{j} \otimes \xi_{j}=A^{i} \wedge B^{j} \otimes\left[\xi_{i}, \xi_{j}\right]\right)$ is to be compared with our $x$ product. The gauge covariant derivative $\mathbb{D}(\mathbb{D}=d+A \wedge, A$ being the vector potential one-form), the contractions $i_{X}$, and the covariant Lie derivative $\mathbb{L}_{X}=i_{X} \mathbb{D}+\mathbb{D} i_{X}$ (all acting on the "differential form part" of $A$ ) are derivations in $\Lambda(M, \mathscr{G})$. The derivations generically denoted $\left(\Omega_{I} \circ\right)$ in Sect. 5 are analogous to $\left(F_{I} \circ\right)$, where $F$ is the field strength $F=d A+\frac{1}{2} A \wedge A$. The projection $P$ for gauge theories is the restriction to a sub-group $H$ of $G$. In order that from a derivation $\delta$ on $\Lambda(M, \mathscr{G})$, one obtains $P \delta$ as a derivation on $\Lambda(M, \mathscr{H})$, the system $H \subset G$ must be weakly reductive.

In Sects. 4-6 we worked out the details for the fibration $\left(M^{\left(D_{0}, D_{1}\right)}, \pi, B_{1}, F_{0}\right)$, i.e., for odd base and even fibre. The only thing that changes if one has the opposite case $\left(M^{\left(D_{0}, D_{1}\right)}, \pi, B_{0}, F_{1}\right)$ is the definition of the projection $P$ : in this case one obtains from a hyperform $\varphi$ the components of $\varphi^{P}$ as

$$
\left\langle X_{1} \ldots X_{p}\left|\varphi^{P}\right| \omega_{1} \ldots \omega_{r}\right\rangle=\left\langle X_{1}^{H} \ldots X_{p}^{H}|\varphi| \omega_{1}^{V} \ldots \omega_{r}^{V}\right\rangle .
$$

Thus in all definitions in Sects. 4 to 6 not referring to charts, one only has to exchange $H$ and $V$. The canonical basis (with respect to natural coordinates in $\left.\left(M^{\left(D_{0}, D_{0}\right)}, \pi, B_{1}, F_{0}\right)\right)$ of the tangent space to the bundle is given by $\left\{{ }_{\alpha} D={ }_{\alpha} \partial\right.$ 
$\left.+{ }_{\alpha} \Gamma^{\mu}{ }_{\mu} \partial,{ }_{\mu} \partial\right\}$. The appearance of ${ }_{\alpha} D$ is the reason why we preferred this fibration to the other one. The derivatives ${ }_{\alpha} D$ are used in supersymmetric theories since they anticommute with (a realisation of) the supertranslation operators $Q_{\alpha}$ of the graded Poincaré group. It has been shown [14] that these covariant derivatives stem from a fibred structure existing on Salam-Strathdee superspace: rigid superspace can be regarded as a principal bundle whose base is the supertranslation group and whose structure group is the ordinary translation group (translations in Minkowski space). This situation exactly reflects our fibration with $\theta$ variables as coordinates in the base and $x$ variables as coordinates in the fibres.

We would like to point out that the exterior calculus on $P$-hyperforms provides all the necessary ingredients for a coordinate-free formulation of superfield actions. The superfields one is dealing with are components of differential forms, say $\omega$ generically. The $\omega$ and their exterior derivatives $d \omega$ are building blocks for the action. We emphasize that the action density has to be a $\left(D_{0}, D_{1}\right) P$-hyperform (thus at least one $P$-hyperform is a necessary ingredient of the theory!) and so one cannot avoid introducing an operation to provide the missing "derivative form part," and this operation is the Hodge *.

One should also observe that in working with $P$-hyperforms one throws away all $\theta$ derivatives. This is precisely what occurs in the usual formulation of superfield theories, where the $\theta$ derivatives are obtained via Berezin integration. It is indeed possible to define this as a (local) operation $\int: \Lambda_{p}^{r}(E) \mapsto \Lambda_{p}^{r-1}(E)$. We will come back to this point in a later publication.

There are several hints that constraints introduced both in global and local supersymmetric theories are of geometric origin. For instance, the chiral constraints on a complex scalar superfield $\varphi$ (Wess-Zumino model) can be stated as the vanishing of certain horizontal components of $d \phi$. In supergravity there is still no systematic method to determine which constraints one has to impose on the torsion in order to reduce the number of components of a superfield (see for instance [3]). We speculate that they emerge geometrically by formulating supergravity in terms of supermanifold integrals. This speculation is supported by the observation [15] that one can derive from a Lagrangian four-form on a supermanifold the constraints for $N=1$ supergravity as part of the field equations. The problem in this approach, namely the lack of invariance under (super)diffeomorphisms, could be overcome by choosing a $(4,4) P$-hyperform as the Lagrangian. The supermanifold integration and the exterior calculus of hyperforms also provide a geometric foundation for the rheonomy conditions in the group manifold approach to supergravity [12]. A further application we have in mind is the formulation of locally supersymmetric Yang-Mills type theories for gravitation and, more generally, of graded Poincaré gauge theories.

Finally, we hope to have convinced Bryce de Witt that Berezin's approach to supermanifold integration is a first step towards a de Rham theory; see his sceptical comments on p. 121 of [4]. We are also pleased to assure Tullio Regge that the "formalism and symbolism" attitude taken by workers in "superphysics" may not be far from acquiring a concrete status; see his remarks on p. 946 in [16]. 


\section{References}

1. Salam, A., Strathdee, J.: Super-Gauge transformations. Nucl. Phys. B 76, 477-482 (1974)

2. Berezin, F.A.: The method of second quantization. New York: Academic Press 1976

3. Wess, J., Bagger, J.: Supersymmetry and supergravity. Princeton: Princeton University Press 1983

4. De Witt, B.: Supermanifolds. Cambridge: Cambridge University Press 1984

5. Rogers, A.: A global theory of supermanifolds. J. Math. Phys. 21, 1352-1365 (1980)

6. Jadczyk, A., Pilch, K.: Superspaces and supersymmetries. Commun. Math. Phys. 78, 373-390 (1981)

7. Wieloch, M.: Zwei Strukturen auf Supermannigfaltigkeiten. Diploma Thesis: Freie Universität Berlin 1982

8. Hoyos, J., Quirós, M., Ramírez Mittelbrunn, J., de Urríes, F.J.: Generalised supermanifolds. J. Math. Phys. 25, 833-854 (1984)

9. Berezin, F.A.: Differential forms on supermanifolds. Sov. J. Nucl. Phys. 30, 605-609 (1979)

10. Zumino, B.: Superspace. In: Unification of the Fundamental Interactions. Ferrara, J., Ellis, J., van Nieuwenhuizen, P. (eds.). New York, London: Plenum Press 1980

11. Rogers, A.: Integration on supermanifolds. In: Mathematical aspects of superspace. Seifert, H.J., Clarke, C.J.S., Rosenblum, A. (eds.). Dordrecht: D. Reidel 1984

12. Ne'eman, Y.: Supermanifolds and Berezin's new integral. Tel Aviv University preprint TAUP N153-83, to appear in the Proceedings of the XII International Conference on Differential Geometric Methods in Theoretical Physics (Clausthal 1983)

13. Rogers, A.: Consistent superspace integration. J. Math. Phys. 26, 385-392 (1985)

14. Aldaya, V., Azcarraga, J.A.: A note on the meaning of covariant derivatives in supersymmetry. Trieste preprint IC/84/40 (1984)

15. Bruzzo, U., Cianci, R.: Structure of supermanifolds and supersymmetry transformations. Commun. Math. Phys. 95, 393-400 (1984)

16. Regge, T.: Group manifold approach to unified gravity. In: Relativity, groups and topology II. De Witt, B., Stora, R. (eds). Amsterdam, Oxford, New York, Tokyo: North-Holland 1984

Communicated by R. Haag

Received April 4, 1985; in revised form May 24, 1985

Note added in proof. After completion of this work we received an article from A. Rogers entitled "On the existence of global integral forms on supermanifolds" (King's College London preprint, 1984), which also deals with Berezin's approach to integration on supermanifolds. What we interpreted as bundle morphisms in a fibre bundle with connection, are interpreted by A. Rogers as restricted transition functions in a subatlas covering the supermanifold. 